\title{
ALKYNYL BROMIDES:
}

1-Bromophenyl acetylene (6a). ${ }^{1} \quad{ }^{1} \mathrm{H}$ NMR $\left(\mathrm{CDCl}_{3}\right)$ : $\delta 7.30(\mathrm{~m}, 3 \mathrm{H}), 7.44(\mathrm{~m}, 2 \mathrm{H}) \cdot{ }^{13} \mathrm{C}$ NMR $\left(\mathrm{CDCl}_{3}\right): 49.7,80.0,122.7,128.3,128.6,132.0$.

1-Bromo-2-(p-methylphenyl) acetylene (6b). ${ }^{1}{ }^{1} \mathrm{H} \mathrm{NMR}\left(\mathrm{CDCl}_{3}\right): \delta 2.35(\mathrm{~s}, 3 \mathrm{H}), 7.10$ $(\mathrm{d}, 2 \mathrm{H}, \mathrm{J}=7.6 \mathrm{~Hz}), 7.32$ (d, 2H, J = 7.6 Hz). ${ }^{13} \mathrm{C} \mathrm{NMR}\left(\mathrm{CDCl}_{3}\right): 21.5,48.8,80.2,119.6$, 129.1, 131.7, 136.7 .

1-Bromo-2-(1-cyclohexenyl) acetylene(6c). ${ }^{2}{ }^{1} \mathrm{H} \mathrm{NMR}\left(\mathrm{CDCl}_{3}\right): \delta 1.60$ (m, 4H), 2.08 (m, 4H), $6.95(\mathrm{~m}, 1 \mathrm{H}) .{ }^{13} \mathrm{C} \mathrm{NMR}\left(\mathrm{CDCl}_{3}\right)$ : 21.3, 22.2, 25.6, 28.8, 46.2, 81.8 .

1-Bromo-2-(1-chlropropyl) acetylene (6d). ${ }^{1}{ }^{1} \mathrm{H} \mathrm{NMR}\left(\mathrm{CDCl}_{3}\right): \delta 1.98(\mathrm{~m}, 2 \mathrm{H}), 2.4(\mathrm{t}$, $2 \mathrm{H}, J=6.75 \mathrm{~Hz}), 3.64$ ( $\mathrm{t}, 2 \mathrm{H}, \mathrm{J}=6.25 \mathrm{~Hz}) .{ }^{13} \mathrm{C} \mathrm{NMR}\left(\mathrm{CDCl}_{3}\right): 17.1,31.0,37.0,43.4$, 78.4.

1-Bromo-1-Octyne (6e). ${ }^{3}{ }^{1} \mathrm{H} \mathrm{NMR}\left(\mathrm{CDCl}_{3}\right): \delta 0.88(\mathrm{t}, 3 \mathrm{H}, \mathrm{J}=8.50 \mathrm{~Hz}), 1.25-1.50(\mathrm{~m}$, $8 \mathrm{H}), 2.19(\mathrm{t}, 2 \mathrm{H}, \mathrm{J}=7.5 \mathrm{~Hz}) .{ }^{13} \mathrm{C} \mathrm{NMR}\left(\mathrm{CDCl}_{3}\right): 14.0,19.7,22.5,28.3,28.5,31.3$, 37.4, 80.4 .

\section{ALKENYL BROMIDES}

(E)- $\beta$-Bromostyrene (4a). ${ }^{4}{ }^{1} \mathrm{H}$ NMR $\left(\mathrm{CDCl}_{3}\right): \delta 6.77(1 \mathrm{H}, \mathrm{d}, \mathrm{J}=14.0 \mathrm{~Hz}), 7.11(\mathrm{~d}, 1 \mathrm{H}$, $J=14.0 \mathrm{~Hz}), 7.26-7.30(\mathrm{~m}, 5 \mathrm{H}) .{ }^{13} \mathrm{C} \mathrm{NMR}\left(\mathrm{CDCl}_{3}\right): \delta 106.5,126.0,128.2,128.7,135.9$, 137.1. 
(Z)- $\beta$-Bromostyrene (4b). ${ }^{4} \quad{ }^{1} \mathrm{H}$ NMR $\left(\mathrm{CDCl}_{3}\right): \delta 6.42(\mathrm{~d}, 1 \mathrm{H}, J=8.25 \mathrm{~Hz}), 7.05(\mathrm{~d}, 1 \mathrm{H}$, $J=8.25 \mathrm{~Hz}), 7.27-7.39(\mathrm{~m}, 3 \mathrm{H}), 7.63-7.66(\mathrm{~m}, 2 \mathrm{H}) .{ }^{13} \mathrm{C} \mathrm{NMR}\left(\mathrm{CDCl}_{3}\right): \delta 106.3,126.1$, 128.2, 128.7, 135.9, 137.1.

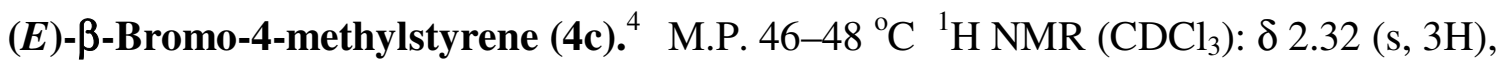
$6.58(\mathrm{~d}, 1 \mathrm{H}, J=14.0 \mathrm{~Hz}), 6.75(\mathrm{~d}, 1 \mathrm{H}, J=14.0 \mathrm{~Hz}), 7.09-7.19(\mathrm{~m}, 4 \mathrm{H}) .{ }^{13} \mathrm{C} \mathrm{NMR}$ $\left(\mathrm{CDCl}_{3}\right): \delta 21.2,105.4,126.0,129.5,133.2,137.0,138.2$.

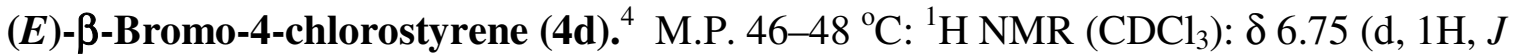
$=14 \mathrm{~Hz}), 7.05(\mathrm{~d}, 1 \mathrm{H}, J=14.0 \mathrm{~Hz}), 7.19(\mathrm{~d}, 2 \mathrm{H}, J=8.4 \mathrm{~Hz}), 7.28(\mathrm{~d}, 2 \mathrm{H}, J=8.4 \mathrm{~Hz}) .{ }^{13} \mathrm{C}$ $\operatorname{NMR}\left(\mathrm{CDCl}_{3}\right): \delta 107.2,127.2,129.0,134.0,134.3,135.9$.

(E)-1-Bromonon-1-ene (4e). ${ }^{4}{ }^{1} \mathrm{H}$ NMR $\left(\mathrm{CDCl}_{3}\right): \delta$ 0.85-0.9 (m, 3H), 1.26-153 (m, 10H), 2.01-2.22 (m, 2H), 5.97-6.19 (m, 2H). ${ }^{13} \mathrm{C} \mathrm{NMR}\left(\mathrm{CDCl}_{3}\right)$ : $\delta$ 14.0, 22.6, 28.1, 29.1, $29.7,31.8,32.9,107.5,136.3$.

\section{ARYLBROMIDES}

Bromobenzene(2a). ${ }^{5}{ }^{1} \mathrm{H}$ NMR $\left(\mathrm{CDCl}_{3}\right): \delta 7.13-7.23(\mathrm{~m}, 3 \mathrm{H}), 7.43-7.47(\mathrm{~m}, 2 \mathrm{H}) .{ }^{13} \mathrm{C}$ $\operatorname{NMR}\left(\mathrm{CDCl}_{3}\right): \delta 122.5,126.6,130.0,131.5$.

4-Bromoanisole(2b). ${ }^{5}{ }^{1} \mathrm{H}$ NMR $\left(\mathrm{CDCl}_{3}\right): \delta 3.77$ (s, 3H), $6.76(\mathrm{~d}, 2 \mathrm{H}, J=7.4 \mathrm{~Hz}), 7.36$ $(\mathrm{d}, 2 \mathrm{H}, \mathrm{J}=7.4 \mathrm{~Hz}) .{ }^{13} \mathrm{C} \mathrm{NMR}\left(\mathrm{CDCl}_{3}\right): \delta 55.4,112.8,115.7,132.2,158.7$.

2-Bromotoluene(2c). ${ }^{6}{ }^{1} \mathrm{H}$ NMR $\left(\mathrm{CDCl}_{3}\right): \delta 2.39(\mathrm{~s}, 3 \mathrm{H}), 7.00-7.06(\mathrm{~m}, 1 \mathrm{H}), 7.16-$ 7.25(m, 2H), 7.50(d, 2H, J = 8.0 Hz). ${ }^{13} \mathrm{C}$ NMR $\left(\mathrm{CDCl}_{3}\right): \delta 23.5,126.6,127.4,127.7$, 136.1, 136.2. 
1-Bromo-2,6-dimethylbenzene(2d). ${ }^{6}{ }^{1} \mathrm{H} \mathrm{NMR}\left(\mathrm{CDCl}_{3}\right): \delta 2.39$ (s, 6H), $7.04(\mathrm{~m}, 3 \mathrm{H})$;

${ }^{13} \mathrm{C} \mathrm{NMR}\left(\mathrm{CDCl}_{3}\right): \delta 23.6,126.5,127.6,128.1,136.2$.

4-Bromochlorobenzene(2e). ${ }^{5}$ M.P. $65-67{ }^{\circ} \mathrm{C} .{ }^{1} \mathrm{H}$ NMR $\left(\mathrm{CDCl}_{3}\right): \delta 7.1-7.22(\mathrm{~m}, 2 \mathrm{H})$, 7.37-7.42 (m, 2H). ${ }^{13} \mathrm{C} \mathrm{NMR}\left(\mathrm{CDCl}_{3}\right): \delta 120.2,130.1,132.7,133.1$.

1,4-Dibromobenzene (2f). ${ }^{5}$ M.P. $86-88{ }^{\circ} \mathrm{C} .{ }^{1} \mathrm{H}$ NMR $\left(\mathrm{CDCl}_{3}\right): \delta 7.34$ (s, $\left.4 \mathrm{H}\right) \cdot{ }^{13} \mathrm{C}$ $\operatorname{NMR}\left(\mathrm{CDCl}_{3}\right): \delta 121.1,133.1$.

1-Bromonaphthalene (2g). ${ }^{6}{ }^{1} \mathrm{H}$ NMR $\left(\mathrm{CDCl}_{3}\right): \delta 7.24(\mathrm{t}, 1 \mathrm{H}, J=8.0 \mathrm{~Hz}), 7.43-7.53(\mathrm{~m}$, 1H), 7.69-7.72 (m, 4H), $8.22(\mathrm{~d}, 1 \mathrm{H}, \mathrm{J}=7.4 \mathrm{~Hz}) ;{ }^{13} \mathrm{C} \mathrm{NMR}\left(\mathrm{CDCl}_{3}\right): \delta 122.7,126.1$, $126.6,127.0,127.2,127.6,128.2,129.8,131.7,134.5$.

3-Bromothiophene(2h). ${ }^{6}{ }^{1} \mathrm{H}$ NMR $\left(\mathrm{CDCl}_{3}\right): \delta 6.99$ (m, 1H), 7.16-7.22(m, 2H); ${ }^{13} \mathrm{C}$ $\operatorname{NMR}\left(\mathrm{CDCl}_{3}\right): \delta$ 110.0, 122.7, 126.6, 129.7.

\section{References:}

1 Yeh, M. C.; Knochel, P.; Tetrahedron Lett. 1989, 30, 4799.

2. Bouyssi, D.; Gore, J.; Balme, G.; Tetrahedron letters 1992, 33, 2811.

3. Correia, J. J. Org. Chem. 1992, 57, 4555.

4. Kuang, C.; Senboku, H.; Tokuda, M. Tetrahedron 2002, 58, 1491.

5. Thiebes, C.; Prakash, G. K. S.; Petasis, N.A.; Olah, G. A. Synlett, 1998, 141.

6. Kitagawa, H.; Shibata, T.; Matsuo, J.; Mukaiyama, T. Bull. Chem. Soc. Jpn. 2002, 75, 339. 


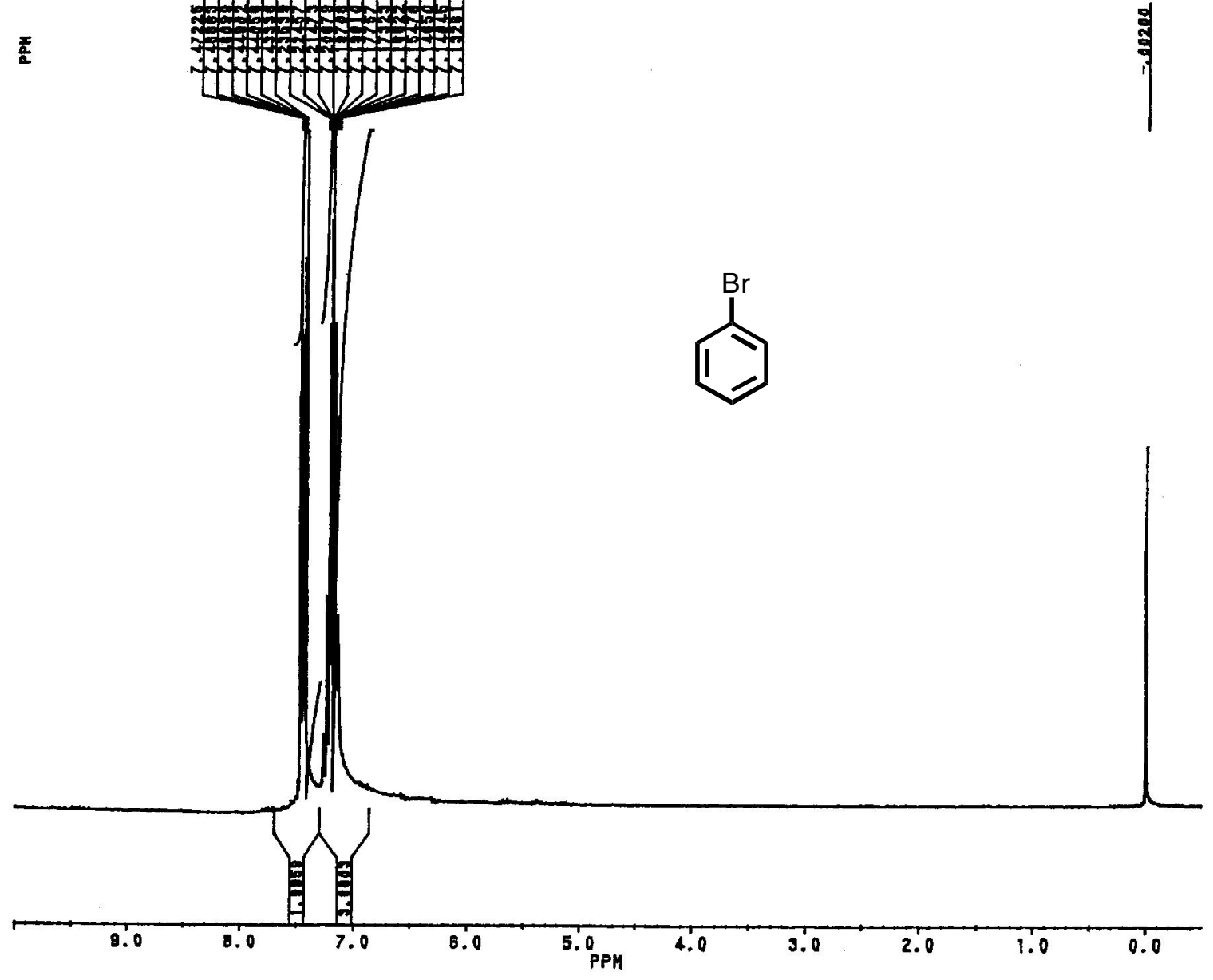


혼
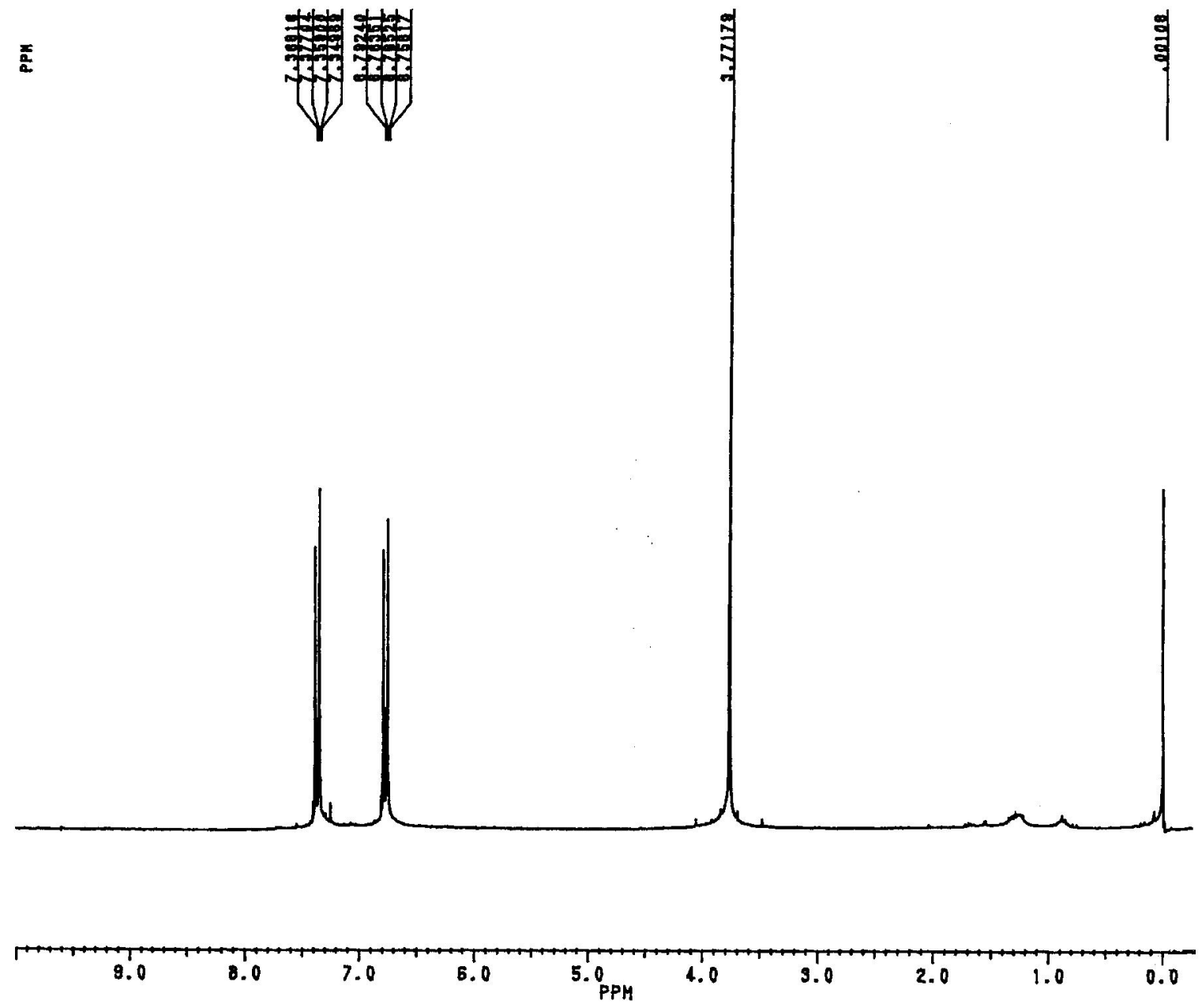
$\overbrace{}^{\mathrm{Br}}$
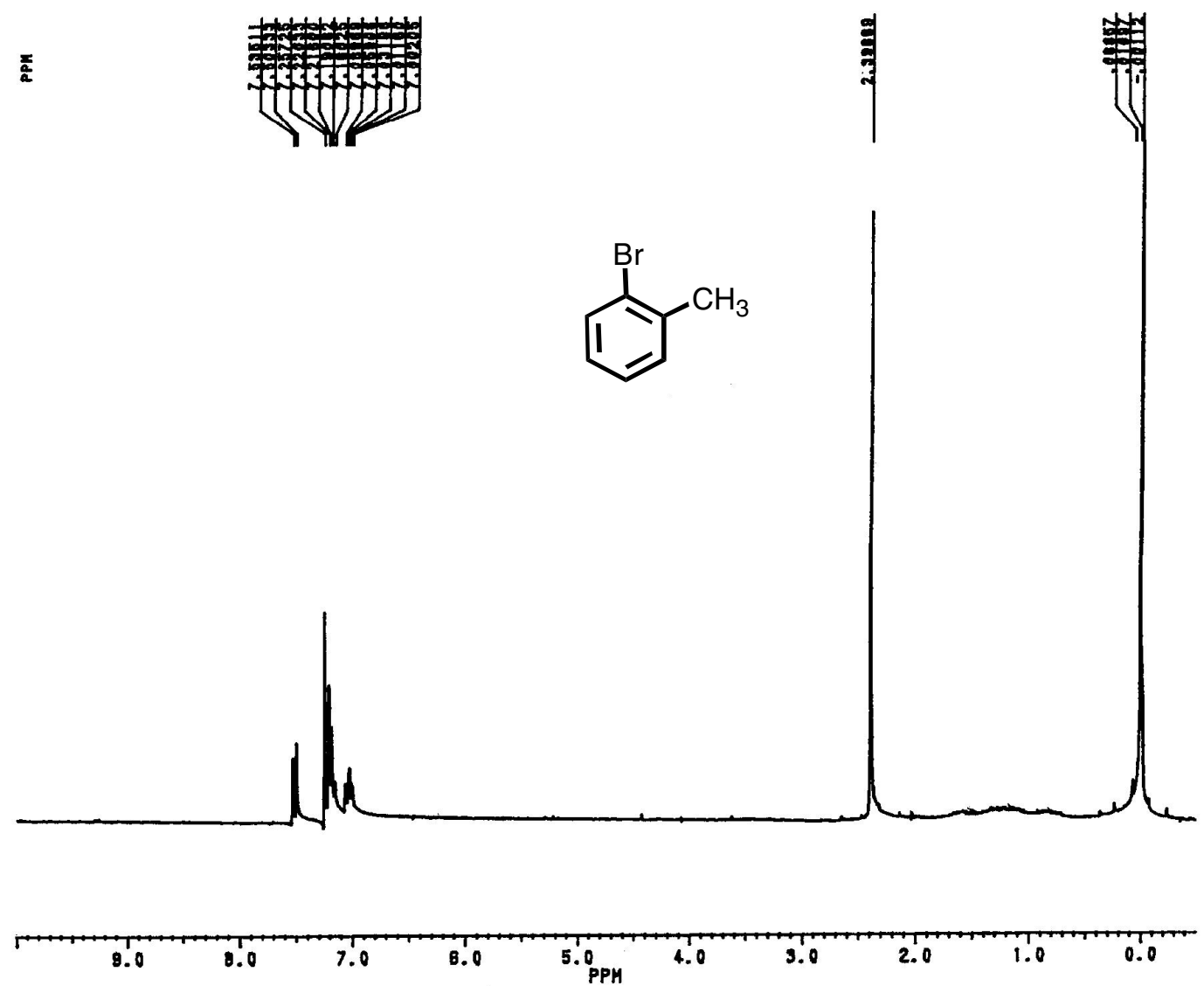
$\underbrace{\mathrm{Br}}_{1} \mathrm{CH}_{3}$ 


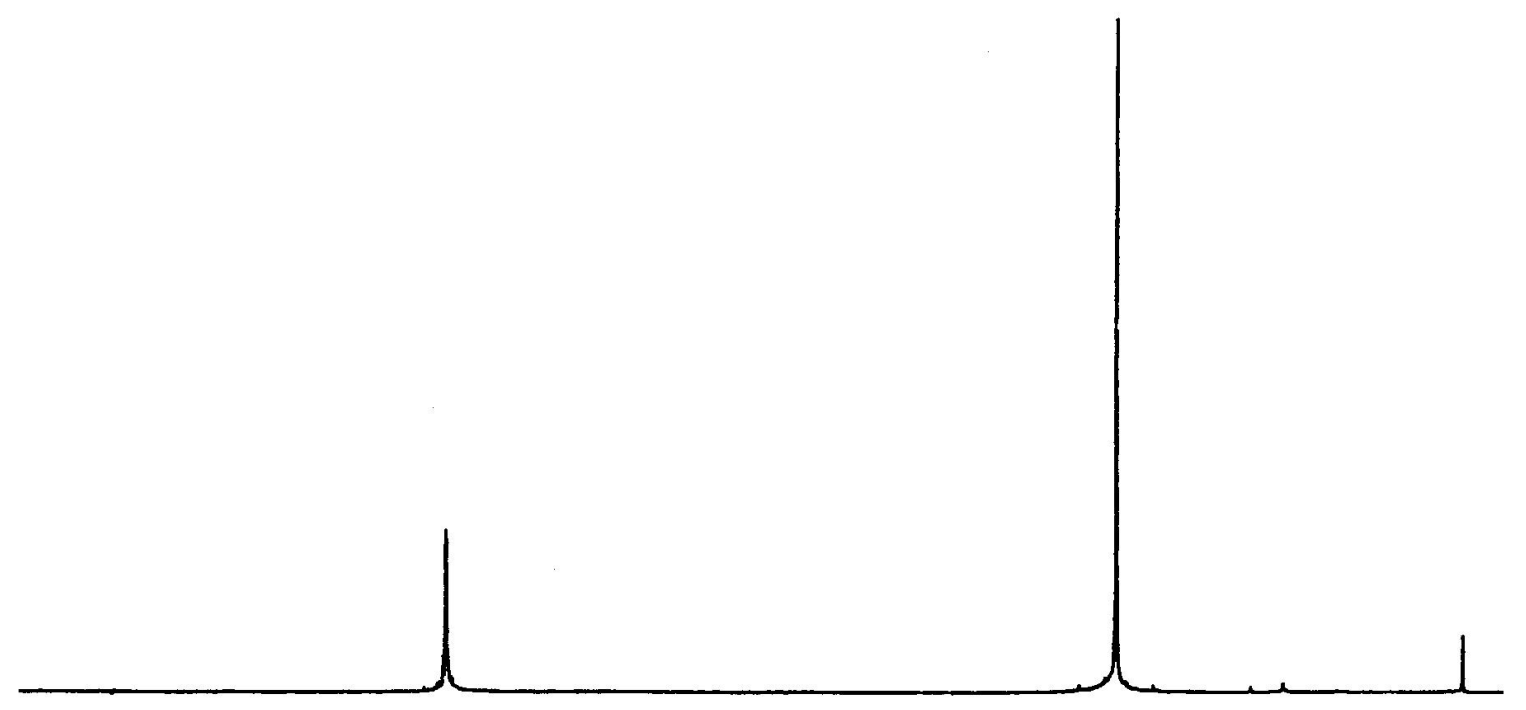

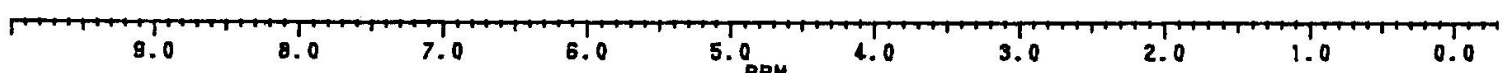


<smiles>Clc1ccc(Br)cc1</smiles>

홀
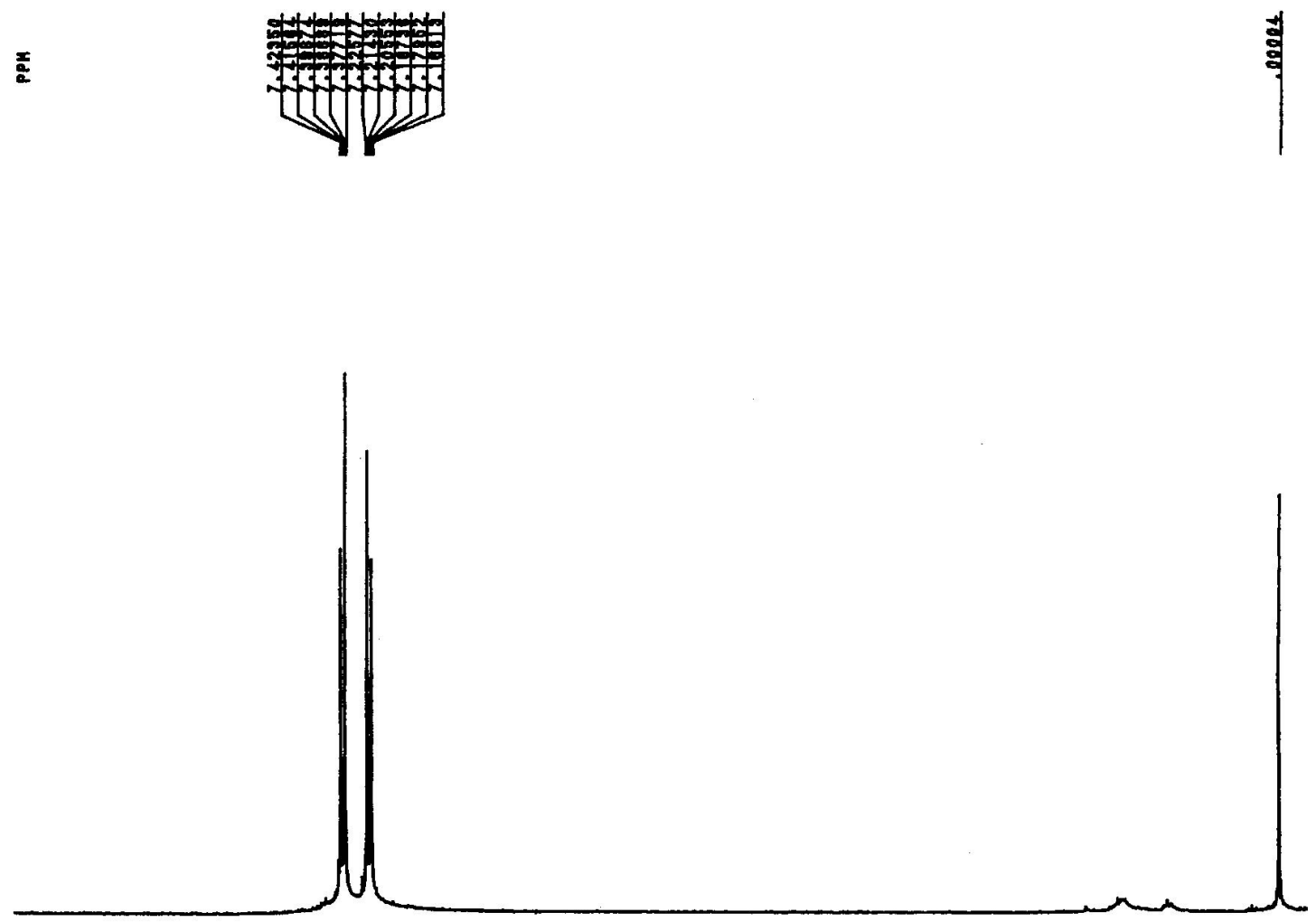

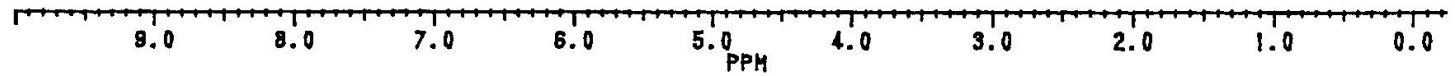



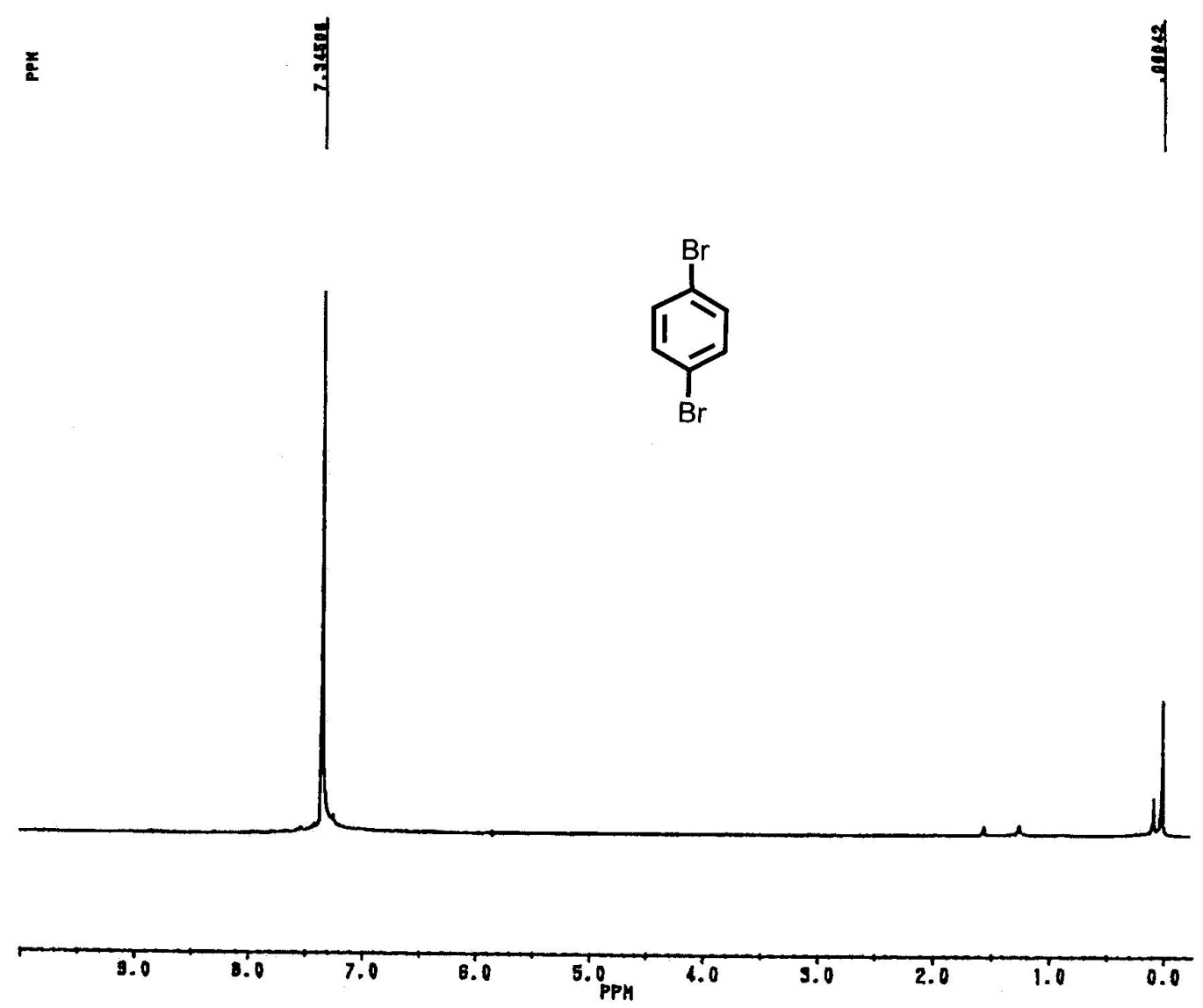


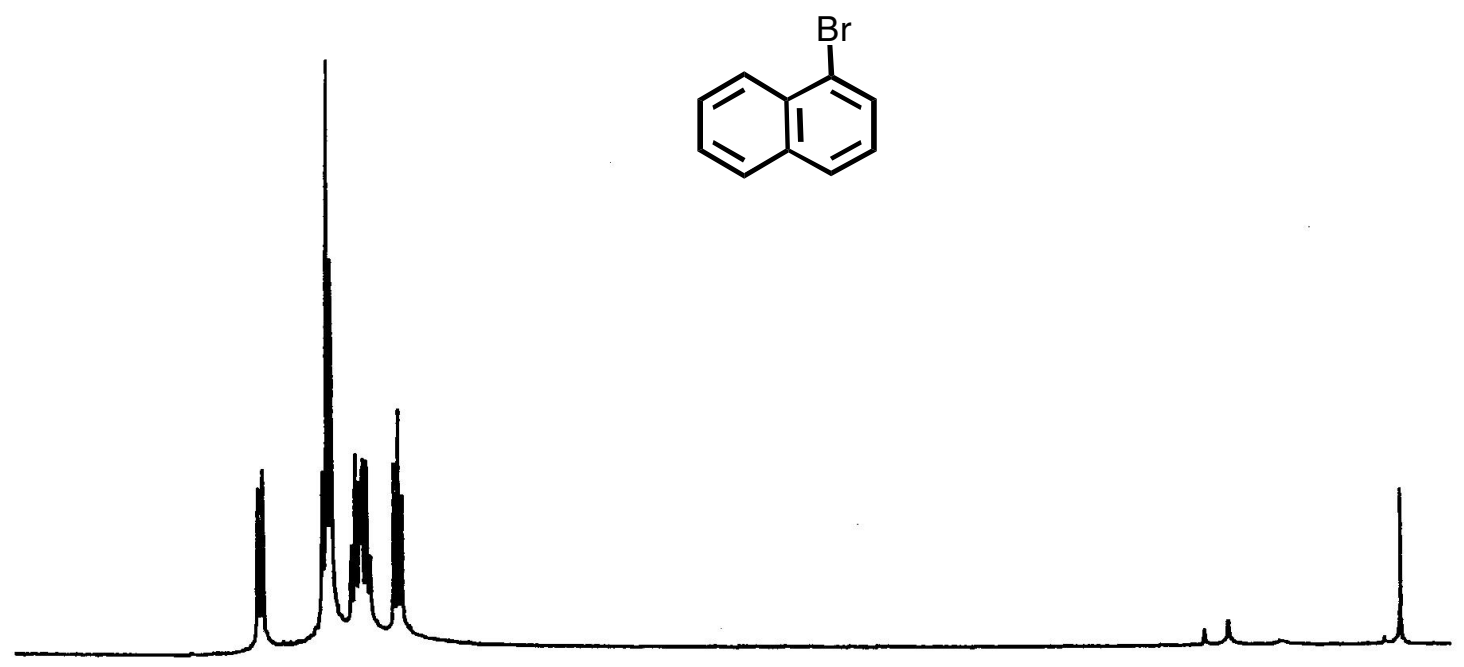

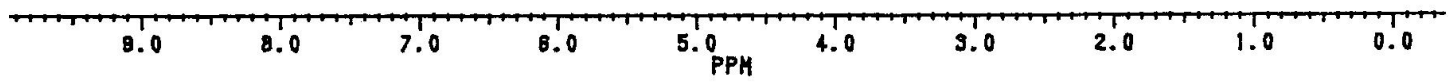



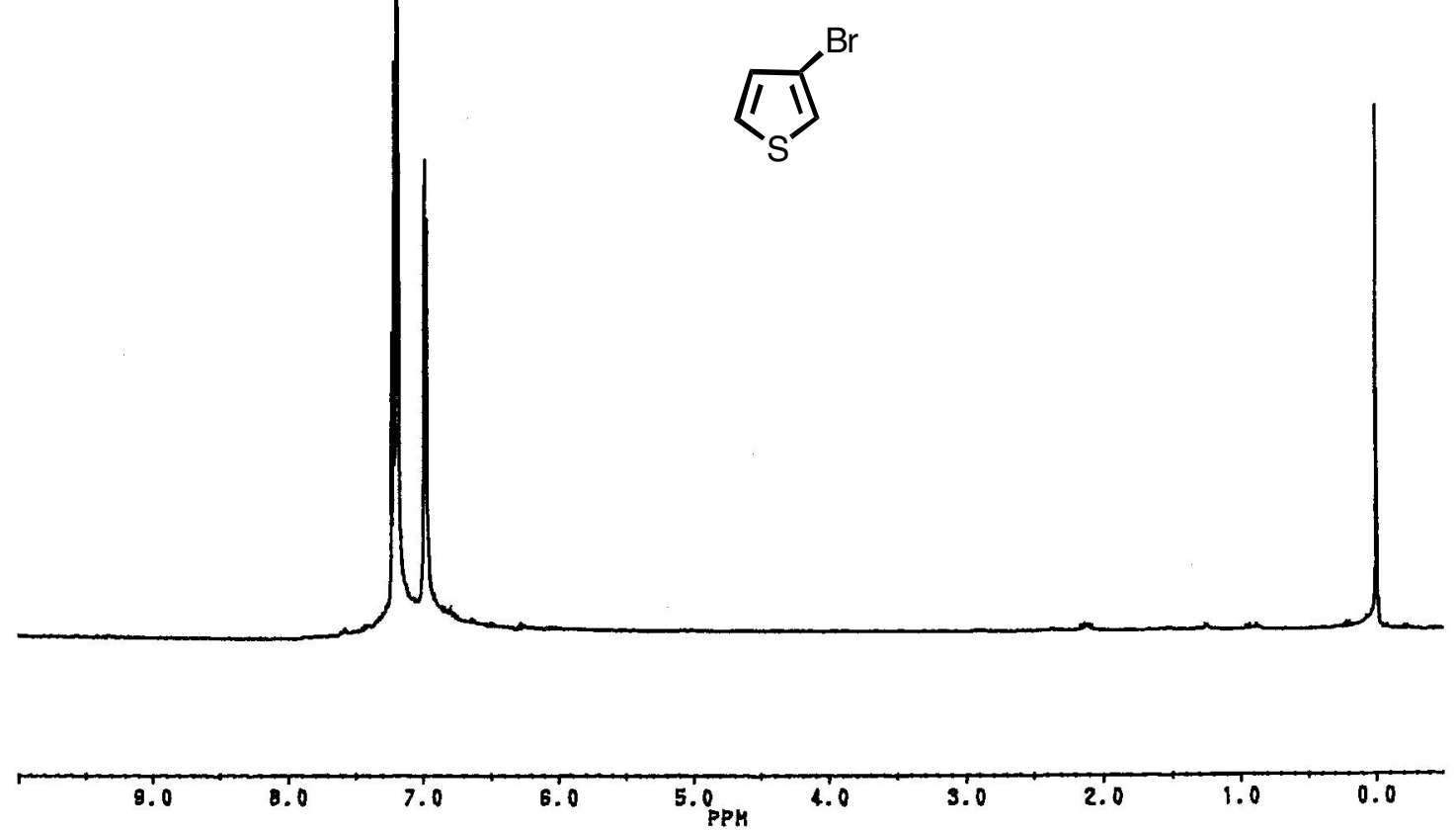


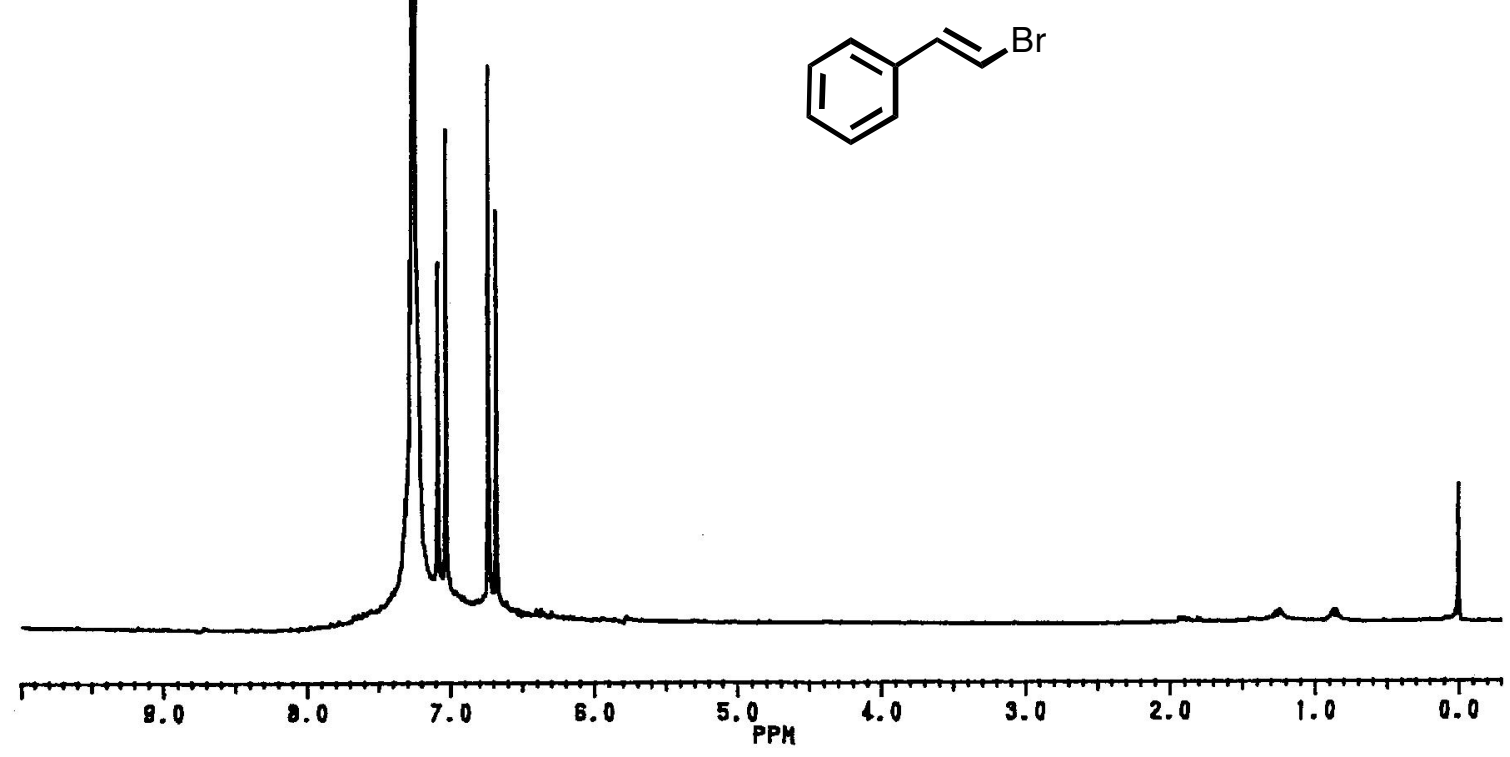



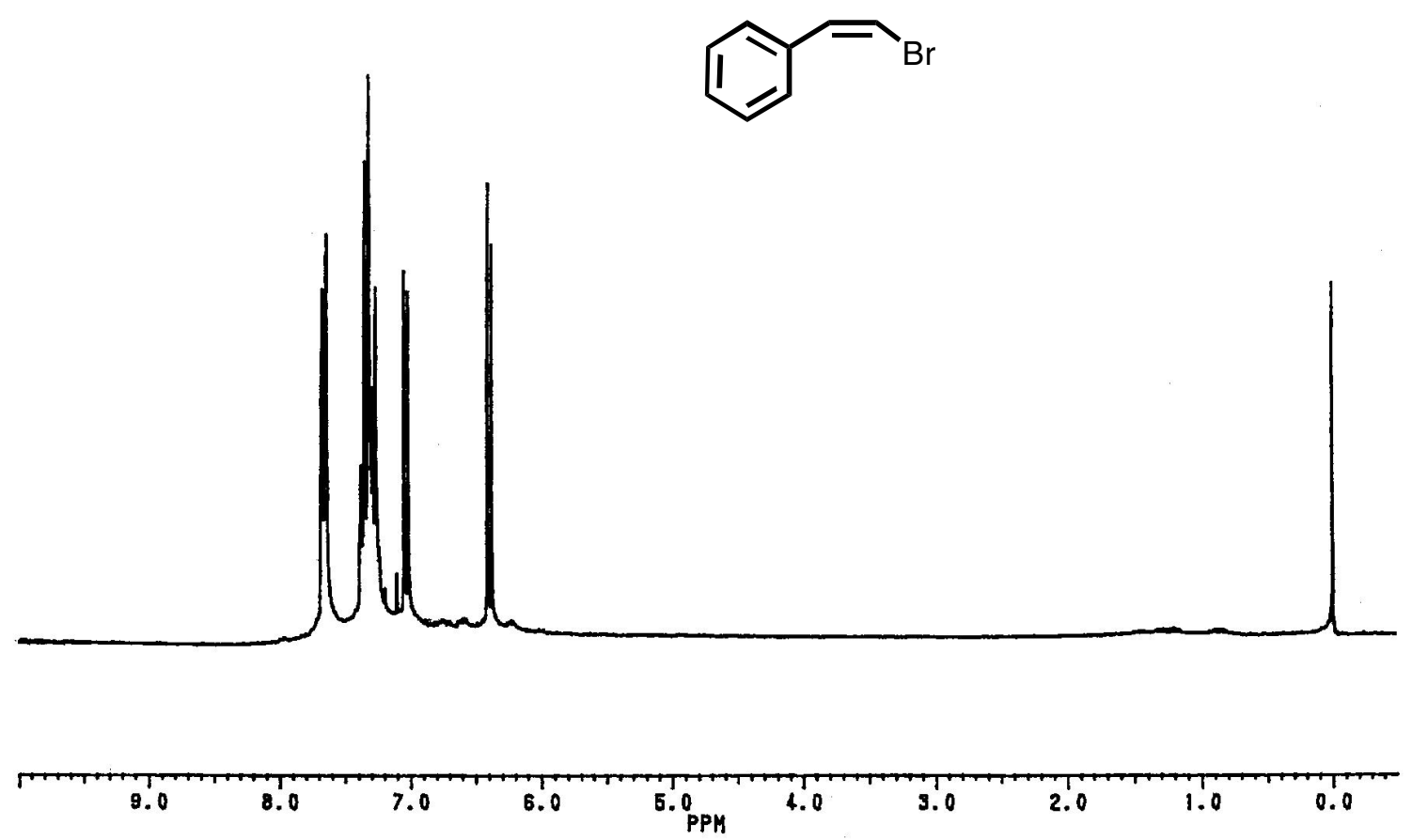

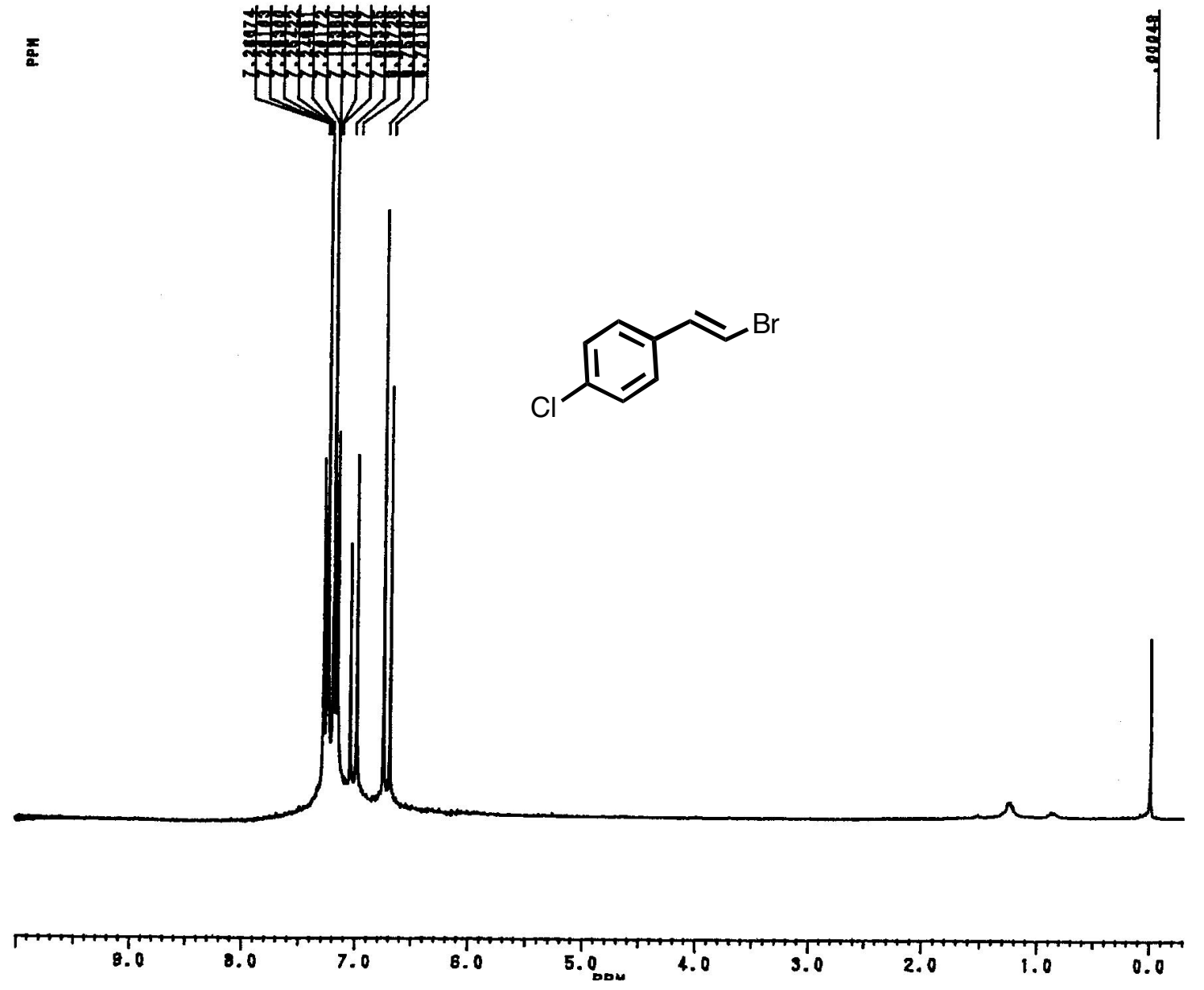

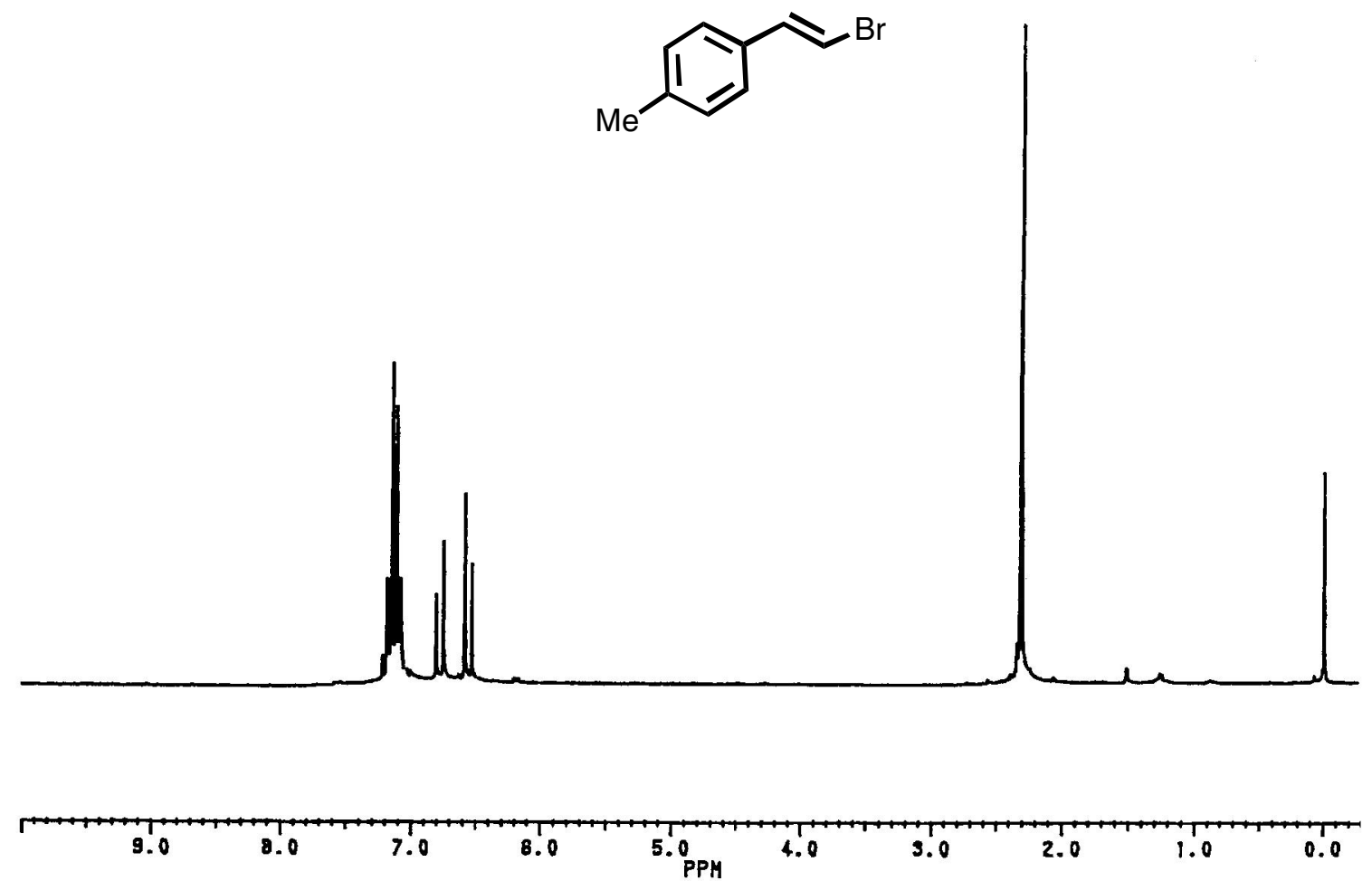

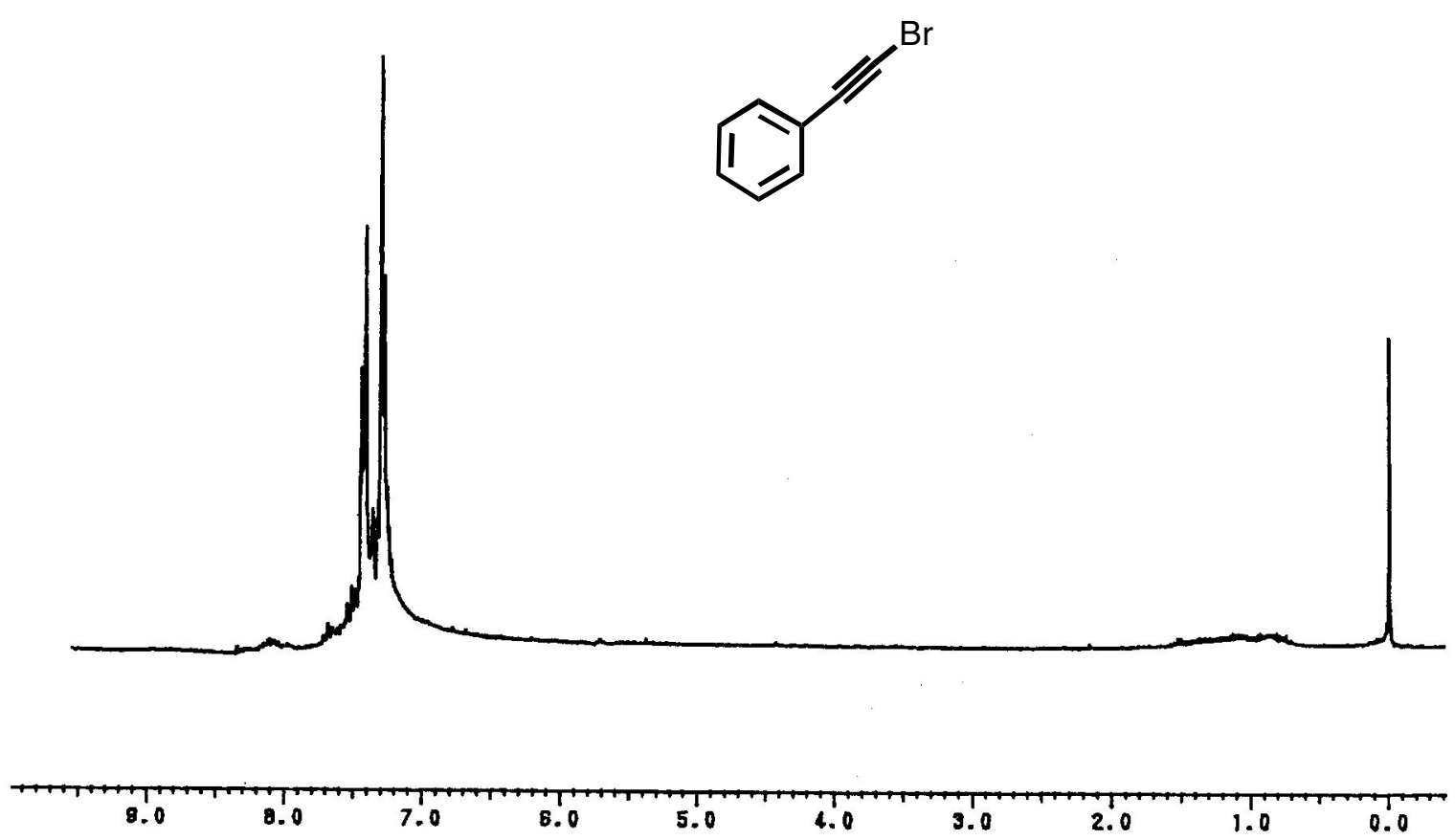

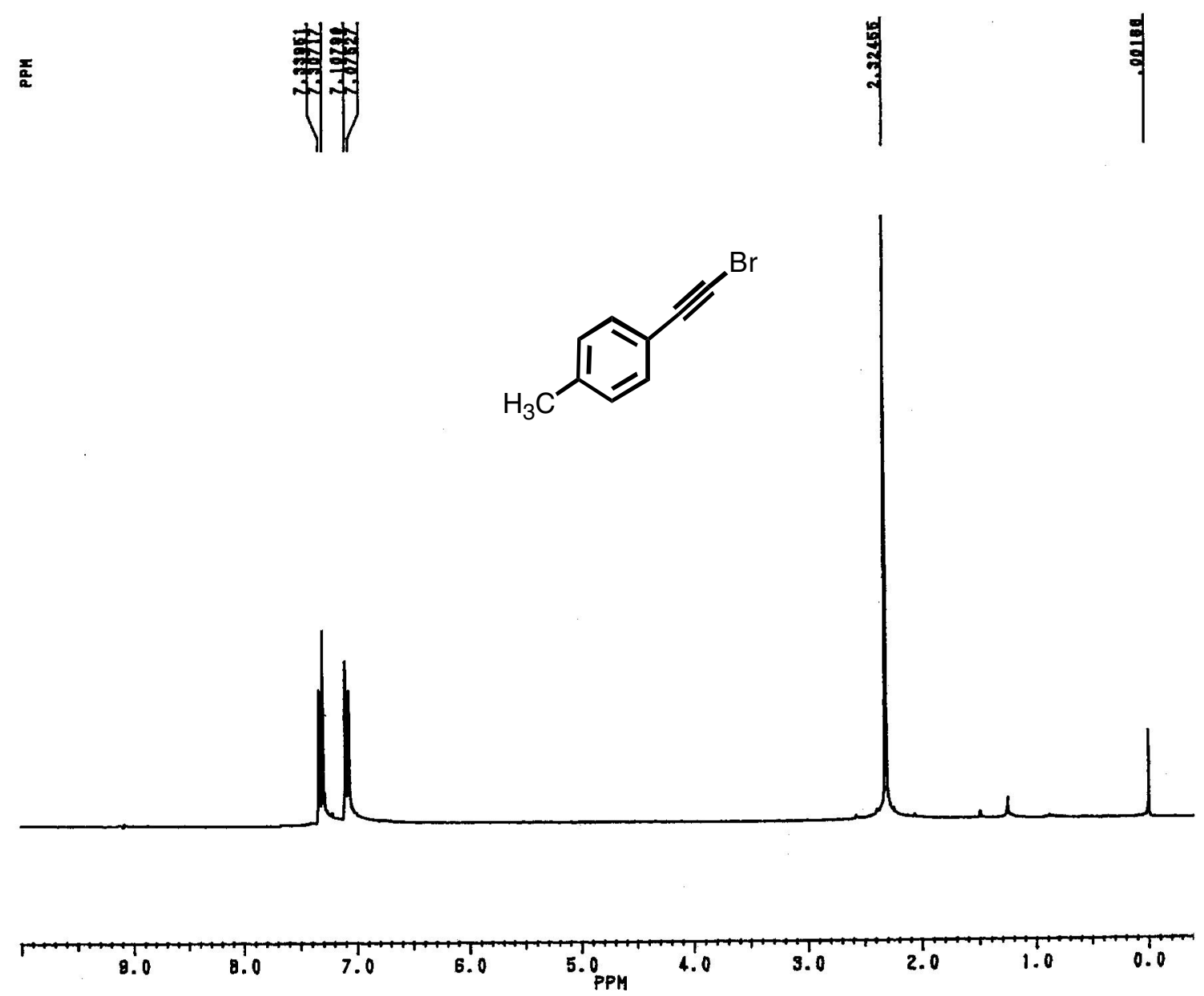


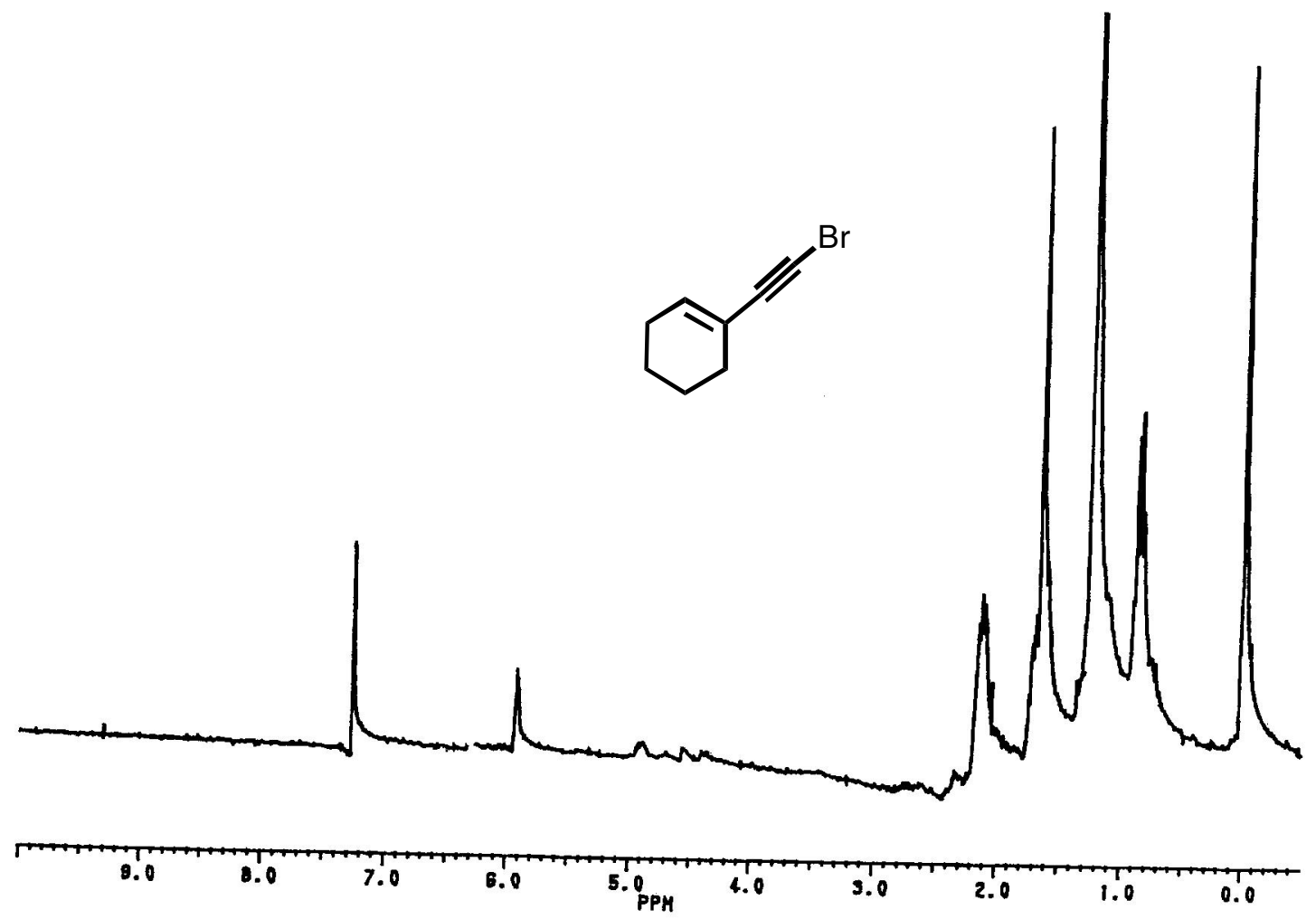




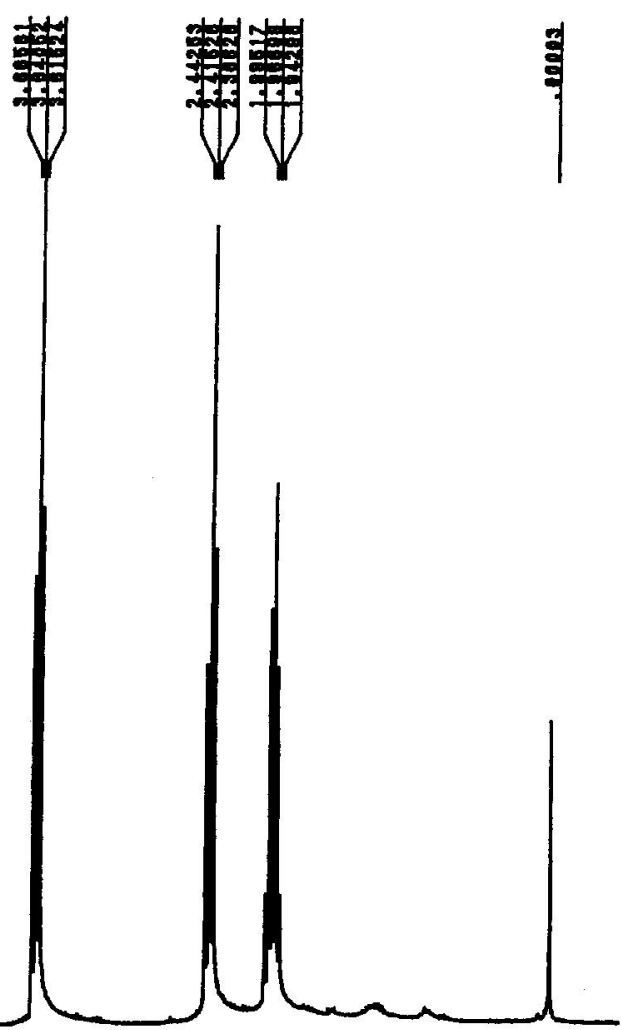

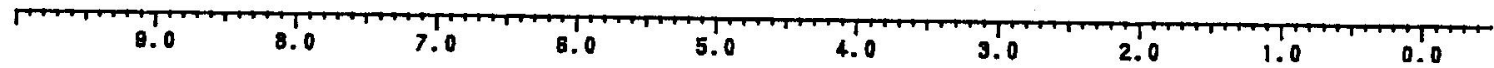



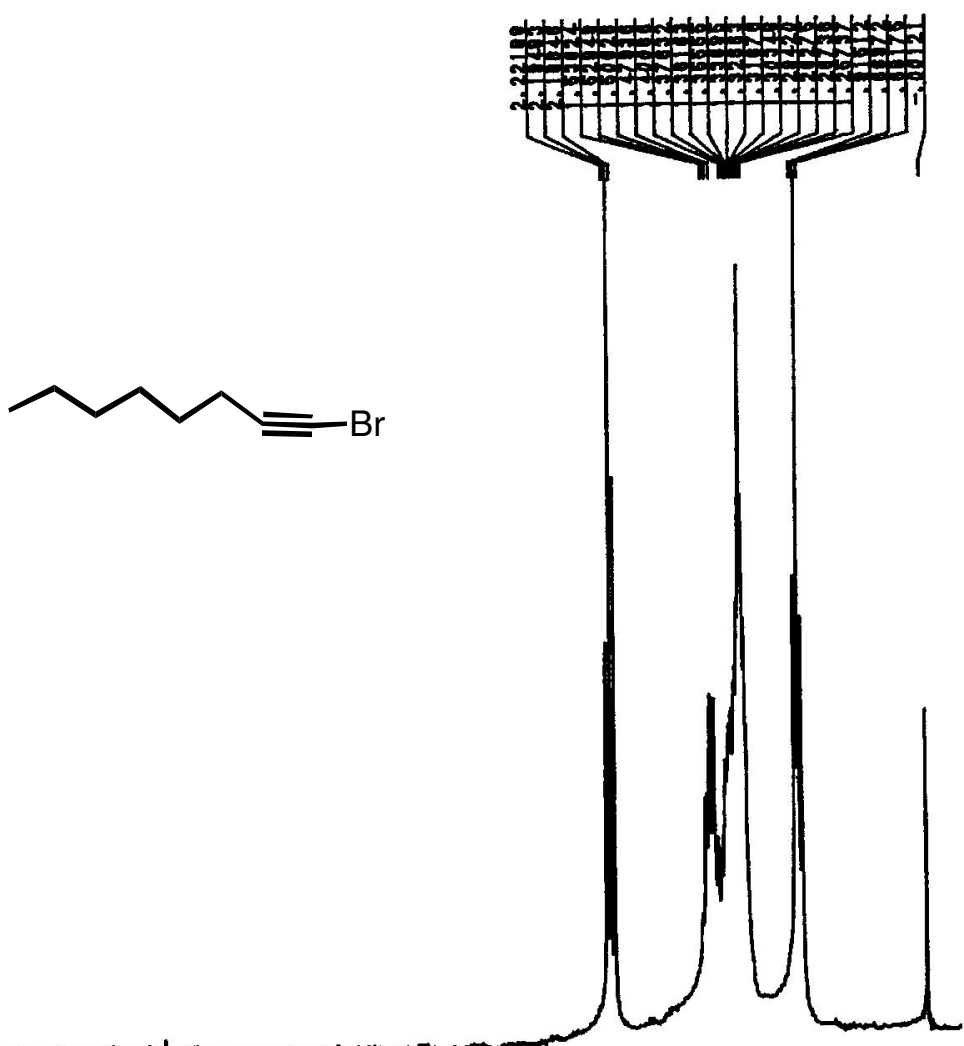

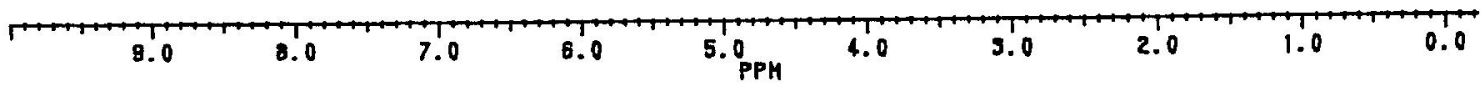

